\title{
Modeling of Gold Circular Sub-Wavelength Apertures on a Fiber Endface for Refractive Index Sensing
}

\author{
Huy NGUYEN ${ }^{1}$, Gregory W. BAXTER ${ }^{1 *}$, Stephen F. COLLINS ${ }^{1}$, \\ Fotios SIDIROGLOU ${ }^{1}$, Ann ROBERTS ${ }^{2}$, and Timothy J. DAVIS ${ }^{3}$ \\ ${ }^{1}$ School of Engineering and Science, Victoria University, PO Box 14428,Melbourne, Victoria 8001, Australia \\ ${ }^{2}$ School of Physics, The University of Melbourne, Victoria 3010, Australia \\ ${ }^{3}$ CSIRO Materials Science \& Engineering, Private Bag 33, Clayton South MDC, Victoria 3169, Australia \\ *Corresponding author: Gregory W. BAXTERＩE-mail: gregory.baxter@vu.edu.au
}

\begin{abstract}
A finite-difference time-domain approach was used to investigate the excitation of surface plasmons of the circular sub-wavelength apertures on an optical fiber endface. This phenomenon provided the basis of a sensitive liquid refractive index sensor. The proposed sensor is compact and has the potential to be used in biomedical applications, having a sensitivity of $(373 \pm 16) \mathrm{nm}$ per refractive index unit (RIU) as found through the variation of a reflection minimum with the wavelength.
\end{abstract}

Keywords: Optical fiber, surface plasmon resonance, periodic array, refractive index sensing, finite-difference time-domain

\section{Introduction}

A surface plasmon resonance (SPR) is an electromagnetic phenomenon which occurs when light is reflected off a thin metal film (e.g. Ag, Au) deposited on a substrate (e.g. glass, quartz, prism) when the angle of incidence is greater than the angle of total internal reflection (TIR). A fraction of this light energy interacts with the collective oscillation of free electrons in the metal film therefore reducing the reflected light intensity [1]. SPR has been widely demonstrated to be an effective optical technique for many types of interface studies [2]. The unique physical properties of SPR have attracted a lot of attention in recent years in optical biosensing research communities. For instance, if analyte molecules are to bind the immobilized target, the local refractive index (RI) changes, leading to a change in the SPR angle [3]. This change can be monitored in real time by detecting changes in the intensity of the reflected light. Even though sensors currently based on the prism configuration can be relatively small, there has been an attempt to realize SPR in an optical fiber to produce a more compact sensor with remote sensing capabilities.

Light propagating in the fiber core and cladding in the form of modes experiences TIR at the cladding-core and cladding-exterior medium interfaces at different angles. Thus, SPR excitation in a fiber is similar to a prism arrangement via TIR for bulk optics. Over the past few years, many fiber-based SPR sensors have been reported, including SPR sensor configurations with multimode, single mode, and polarization 
maintaining fibers coated with a thin metallic layer [4-7]. More recently, there have been many successful attempts to realize SPR sensors in optical fibers [8-11].

The interaction of light with surface plasmons (SPs) in metallic sub-wavelength apertures has resulted in demonstrations of enhanced optical transmission [12]. The demonstration indicated that transmitted light through sub-wavelength aperture arrays at certain wavelengths had a much higher intensity than that estimated by the classical theory [13]. Recently, due to the impressive progress in the nano-fabrication technology $[14,15]$, many researchers have pursued this idea in a quest to create sensitive sensors by fabricating an array of nanostructures at the end face of an optical fiber, for instance nanorods [16], nanoholes [17], and nanoparticles [18]. However, these early proposals offer preliminary designs with little theoretical or experimental evidence to show that the performance of optical fiber sensors would be enhanced by a metallic nanostructure.

This paper describes the modeling of a pattern of gold sub-wavelength aperture arrays directly fabricated on the end face of an optical fiber and its application as an optical refractive index sensor based on the surface plasmon resonance. The computational reflection data for sub-wavelength apertures as a function of the surrounding refractive index and geometrical parameters will be presented.

\section{Methodology and technique}

The experimental setup to be modeled is shown in Fig. 1. A sensing fiber is connected to one side of a $2 \times 1$ optical fiber coupler. A light source and an optical spectrum analyzer (OSA) are connected to the other two coupler ends. Light is coupled via the coupler into the fiber device, where it interacts with the array of circular apertures. The reflected spectrum, modified by the array, is detected by the OSA. To investigate the effect of changing the refractive index of the medium surrounding the fiber end face, a range of liquid refractive indices (between 1.33 and 1.47) are used.

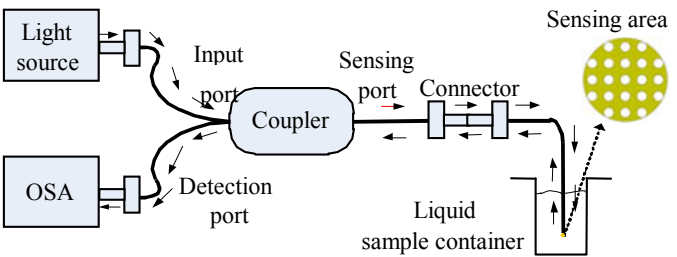

Fig. 1 Experimental arrangement assumed for modeling.

To simulate the optical properties of the circular apertures array assumed to be deposited on the fiber endface, electromagnetic simulations using commercial finite difference time domain (FDTD) software (Lumerical Solution Inc., Canada) [19] were carried out. The incident light was a plane wave propagating along the $z$-direction. Periodic boundary conditions were implemented on the sides, and perfectly matched layers were used to eliminate reflections at the upper and lower surfaces. Figure 2 illustrates an array of $15 \times 15$ circular apertures with the following parameters: $P=500 \mathrm{~nm}, D=300 \mathrm{~nm}$, and $T=140 \mathrm{~nm}$. A unit circular aperture simulated structure and the electric field around the circular aperture are shown in Figs. 3(a) and 3(b). The reflected power was determined by integrating the $z$-component of the Poynting vector over the lower surface. Values were normalized to the incident power. Optical constants for gold were taken from [20].

Initially, the refractive indices for the regions of the optical fiber and the sample were taken as 1.5 and 1 (air). Subsequently, the RI of the sample varied corresponding to a set of liquids or solutions. This enabled the sensitivity of the proposed optical fiber sensor to be explored.

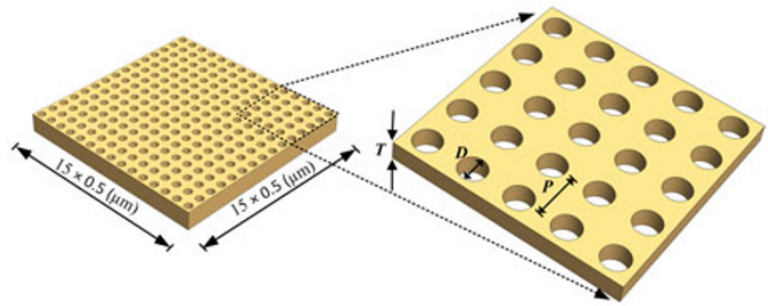

Fig. 2 A $15 \times 15$ gold array of sub-wavelength circular apertures. 


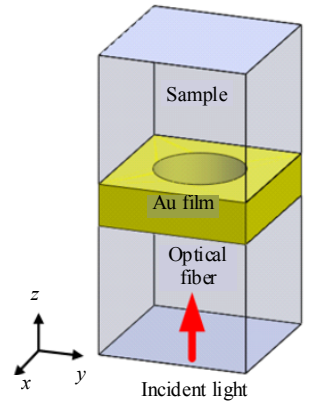

(a)

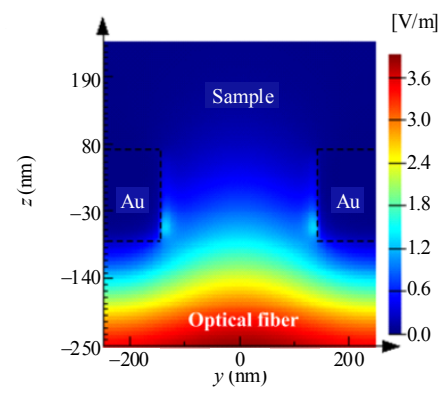

(b)
Fig. 3 Details of a unit cell showing: (a) the circular aperture simulated in FDTD and (b) the intensity of the electric field around the circular aperture (the circular aperture is centered at the origin, and the film boundaries are outlined in dashed lines).

\section{Results and discussion}

Figure 4(a) shows the reflection as a function of

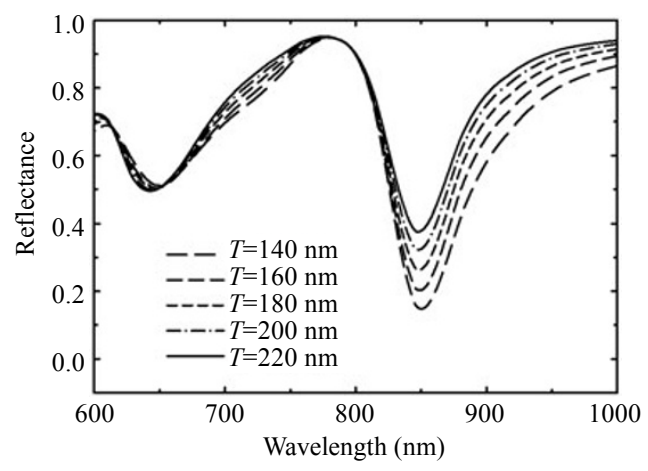

(a)

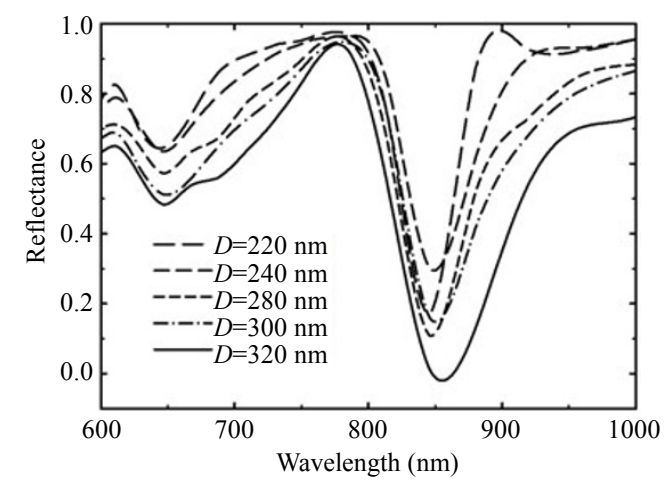

(b)

Fig. 4 FDTD calculations of the reflectance intensity through a circular aperture array with (a) the gold film thickness, $T$, varying from $140 \mathrm{~nm}$ to $220 \mathrm{~nm}$ while $P$ and $D$ remain at $500 \mathrm{~nm}$ and $300 \mathrm{~nm}$, respectively and (b) the circular aperture diameter, $D$, varying from $220 \mathrm{~nm}$ to $320 \mathrm{~nm}$ while $P$ and $T$ remain at $500 \mathrm{~nm}$ and $140 \mathrm{~nm}$, respectively. the wavelength for circular apertures of a fixed periodicity of $500 \mathrm{~nm}$ and a diameter of $300 \mathrm{~nm}$. It can be seen that an increase in the thickness of the metal film leads to a narrower resonant dip and a near-uniform increase in the reflection intensity at longer wavelengths.

Figure 4(b) shows the reflection as a function of the wavelength for circular apertures of a fixed thickness of $140 \mathrm{~nm}$ and a periodicity of $500 \mathrm{~nm}$. It indicates that an increase in the diameter of the circular apertures from $220 \mathrm{~nm}$ to $320 \mathrm{~nm}$ leads to a wider resonant dip wavelength and a non-uniform decrease in the reflection intensity at longer wavelengths.

Figure 5 shows that by increasing the periodicity of the circular apertures from $440 \mathrm{~nm}$ to $520 \mathrm{~nm}$, a red-shift in the resonant dip wavelength is observable from $760 \mathrm{~nm}$ to $880 \mathrm{~nm}$. This wavelength shift represents a contrast to the cases shown in Figs. 4(a) and 4(b).

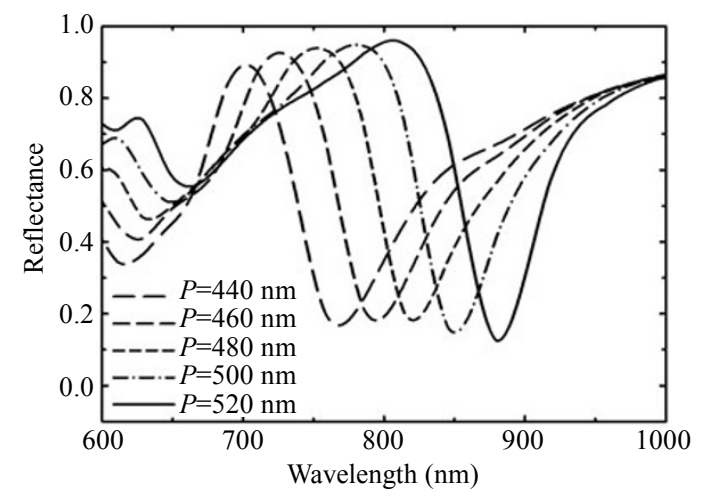

Fig. 5 FDTD calculations of the reflectance intensity through a circular apertures array with the periodicity, $P$, varying from $440 \mathrm{~nm}$ to $520 \mathrm{~nm}$ while $D$ and $T$ remain at $300 \mathrm{~nm}$ and $140 \mathrm{~nm}$, respectively.

These findings suggest that through a suitable choice of parameters (thickness, diameter, and periodicity) a reasonably sharp dip can be obtained for use as a sensing device for tracking the wavelength with the RI. Thus, an array of circular apertures with the following parameters $T=140 \mathrm{~nm}$, $P=500 \mathrm{~nm}$, and $D=300 \mathrm{~nm}$ were selected to explore the sensing capability. As shown in Fig. 6, 
the simulated resonances (reflectance minimum) underwent a red-shift as the index surrounding them increased.

When the minimum dip wavelength for each liquid was plotted against the RI, as shown in Fig.7, a straight line could be fitted to the calculation points. This linear relationship between the shift of minimum in the reflection spectrum $\lambda_{\min }$ and the change in the RI (from 1.33 to 1.47) of the surrounding medium provided a sensitivity of $(373 \pm 16) \mathrm{nm} / \mathrm{RIU}$ (nm/refractive index unit).

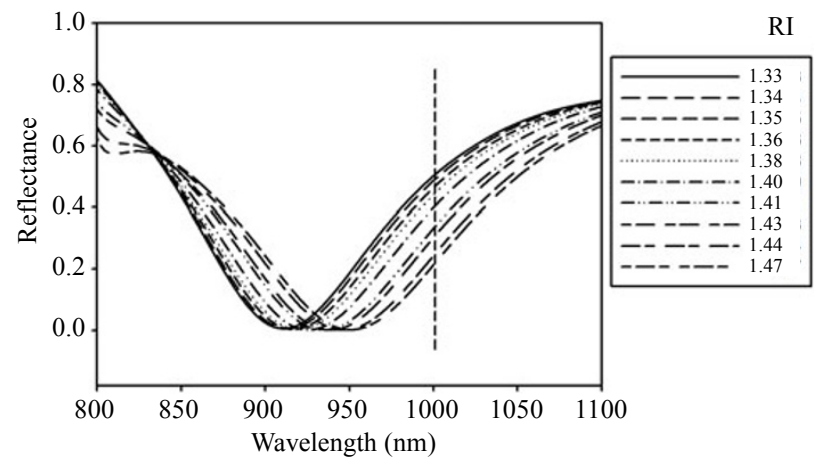

Fig. 6 FDTD calculations of the reflected power spectra for the fiber sensor (circular apertures) in various liquids with the gold film thickness $T=140 \mathrm{~nm}$, periodicity $P=500 \mathrm{~nm}$, and hole diameter $D=300 \mathrm{~nm}$ (the vertical dashed line at $1000 \mathrm{~nm}$ indicates the reflectance values used to obtain Fig. 8).

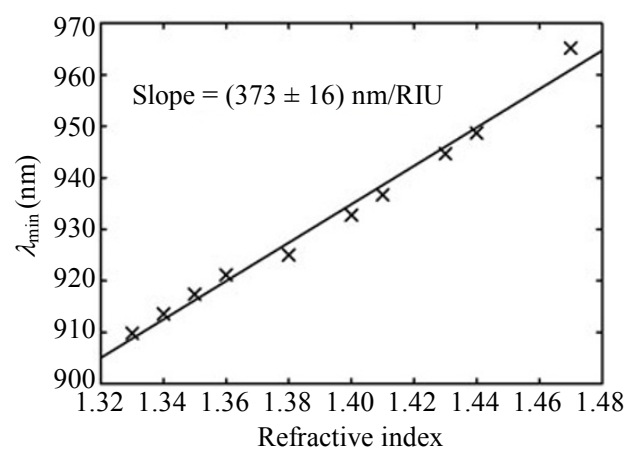

Fig. 7 Dependence of the SPR dip wavelength on the RI of various liquids, showing the linear fit (the circular parameters: $T=140 \mathrm{~nm}, P=500 \mathrm{~nm}$, and $D=300 \mathrm{~nm}$ ).

An alternative form of the RI sensor, which does not involve an OSA, could be realized from the results shown in Fig. 6 by the application of a band-pass filter at around $1000 \mathrm{~nm}$, with the resultant intensities measured using a suitable detector. This is analogous to the conversion of optical fiber Bragg grating [21] wavelengths to intensities through the use of an edge filter [22]. The extracted variation of reflectance with the RI is shown in Fig. 8 and is described well by a linear fit of slope $(-2.73 \pm 0.07)$ normalized reflectance per RIU which is approximately 100 times more sensitive than what would be obtained by simply relying on the Fresnel reflection at the fiber end. In such an arrangement, the filter could be a fiber Bragg grating operating in reflection. Furthermore, as the range over which the reflectance exhibits a considerable variation is greater than $50 \mathrm{~nm}$, such a setup could involve the multiplexing of many sensors [23].

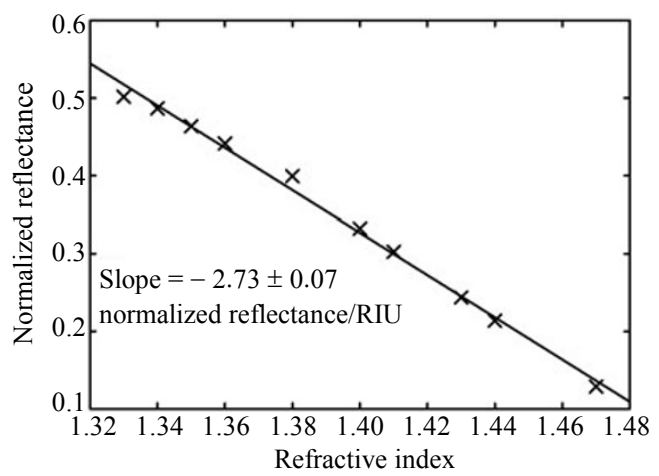

Fig. 8 Dependence of SPR normalized reflectance at a wavelength of $1000 \mathrm{~nm}$, extracted from Fig. 6, on the RI of various liquids, showing the linear fit (the circular parameters: $T$ $=140 \mathrm{~nm}, P=500 \mathrm{~nm}$, and $D=300 \mathrm{~nm})$.

\section{Conclusions}

A study was undertaken to investigate a periodic array of gold nanostructures assumed to be deposited on the endface of an optical fiber. The analysis showed the shifts in dips associated with the optical reflection spectrum of light from circular apertures and found the usefulness of this device as a compact and sensitive liquid refractive index sensor. Compared to similar techniques such as nanorods[16] and nanoparticles [18] which have 
sensitivities of about $180 \mathrm{~nm} / \mathrm{RIU}$ and $196 \mathrm{~nm} / \mathrm{RIU}$, respectively, the proposed sensor showed a much better result of $(373 \pm 16) \mathrm{nm} / \mathrm{RIU}$. More details of the focused ion beam fabrication and experimental processes are available elsewhere [24]. This investigation will assist in designing structures that maximize the sensitivity of a device to small changes in the refractive index.

\section{Acknowledgment}

The authors would like to acknowledge the financial support of Victoria University and the Australian Research Council (project number DP0878268).

Open Access This article is distributed under the terms of the Creative Commons Attribution License which permits any use, distribution, and reproduction in any medium, provided the original author(s) and source are credited.

\section{References}

[1] E. Kretschmann and H. Raether, "Radiative decay of nonradiative surface plasmons excited by light," $Z$. Naturforsch. A, vol. 23, no. 12, pp. 2135-2136, 1968.

[2] D. V. Shankaran, K. V. Gobi, and N. Miura, "Recent advancements in surface plasmon resonance immunosensors for detection of small molecules of biomedical, food and environmental interest," Sensors and Actuators B: Chemical, vol. 121, no. 1, pp. 158-177, 2007.

[3] P. Pattnaik, "Surface plasmon resonance: applications in understanding receptor-ligand interaction," Applied Biochemistry and Biotechnology, vol. 126, no. 2, pp. 79-92, 2005.

[4] R. C. Jorgenson and S. S. Yee, "A fiber-optic chemical sensor based on surface plasmon resonance," Sensors and Actuators B: Chemical, vol. 12, no. 3, pp. 213-220, 1993.

[5] A. Trouillet, C. Ronot-Trioli, C. Veillas, and H. Gagnaire, "Chemical sensing by surface plasmon resonance in a multimode optical fibre," Pure and Applied Optics: Journal of the European Optical
Society Part A, vol. 5, no. 2, pp. 227-237, 1996.

[6] M. Piliarik, J. Homola, Z. Manikova, and J. Ctyroky, "Surface plasmon resonance sensor based on a single-mode polarization-maintaining optical fiber," Sensors and Actuators B: Chemical, vol. 90, no. 1-3, pp. 236-242, 2003.

[7] A. J. C. Tubb, F. P. Payne, R. B. Millington, and C. R. Lowe, "Single-mode optical fiber surface plasma wave chemical sensor," Sensors and Actuators B: Chemical, vol. 41, no. 1-3, pp. 71-79, 1997.

[8] A. K. Sharma, R. Jha, and B. D. Gupta, "Fiber-optic sensors based on surface plasmon resonance: a comprehensive review," IEEE Sensors Journal, vol. 7, no. 8, pp. 1118-1129, 2007.

[9]X. Fan, I. M. White, S. I. Shopova, H. Zhu, J. D. Suter, and Y. Sun, "Sensitive optical biosensors for unlabeled targets: a review," Analytica Chimica Acta, vol. 620, no. 1-2, pp. 8-26, 2008.

[10] O. S. Wolfbeis, "Fiber-optic chemical sensors and biosensors," Analytical Chemistry, vol. 80, no. 12, pp. 4269-4283, 2008.

[11] B. Lee, S. Roh, and J. Park, "Current status of micro- and nano-structured optical fiber sensors," Optical Fiber Technology, vol. 15, no. 3, pp. 209-221, 2009.

[12] T. W. Ebbesen, H. J. Lezec, H. F. Ghaemi, T. Thio, and P. A. Wolff, "Extraordinary optical transmission through sub-wavelength hole arrays," Nature, vol. 391, pp. 667-669, 1998.

[13] H. A. Bethe, "Theory of diffraction by small holes," Physical Review, vol. 66, no. 7-8, pp. 163-182, 1944.

[14] J. N. Anker, W. P. Hall, O. Lyandres, N. C. Shah, J. Zhao, and R. P. Van Duyne, "Biosensing with plasmonic nanosensors," Nature Materials, vol. 7, no. 6, pp. 442-453, 2008.

[15] P. K. Jain and M. A. El-Sayed, "Plasmonic coupling in noble metal nanostructures," Chemical Physics Letters, vol. 487, no. 4-6, pp. 153-164, 2010.

[16] E. J. Smythe, E. Cubukcu, and F. Capasso, "Optical properties of surface plasmon resonances of coupled metallic nanorods," Optics Express, vol. 15, no. 12, pp. 7439-7447, 2007.

[17] A. Dhawan, M. Gerhold, and J. Muth, "Plasmonic structures based on subwavelength apertures for chemical and biological sensing applications," IEEE Sensors Journal, vol. 8, no. 6, pp. 942-950, 2008.

[18] Y. Lin, Y. Zou, and R. G. Lindquist, "A reflection-based localized surface plasmon resonance fiber-optic probe for biochemical 
sensing," Biomedical Optics Express, vol. 2, no. 3, pp. 478-484, 2011.

[19] Lumerical Solutions, Inc., FDTD Solutions User Manual. Vancouver, BC, Canada, 2011.

[20] P. B. Johnson and R. W. Christy, "Optical constants of the noble metals," Physical Review B, vol. 6, no. 12, pp. 4370-4379, 1972.

[21] K. O. Hill and G. Meltz, "Fiber Bragg grating technology fundamentals and overview," Journal of Lightwave Technology, vol. 15, no. 8, pp. 1263-1276, 1997.

[22] S. M. Melle, K. Lui, and R. M. Measures, "Practical fiber-optic Bragg grating strain gauge system,"
Applied Optics, vol. 32, no. 19, pp. 3601-3609, 1993.

[23] H. K. Bal, F. Sidiroglou, S. F. Collins, and Z. Brodzeli, "Multiplexing of fibre optic reflective sensors using Bragg gratings," Measurement Science and Technology, vol. 21, no. 9, pp. 094011 (4 pages), 2010.

[24] H. Nguyen, "Optical fibre surface plasmon resonance sensors based on a metallic array of sub-wavelength apertures," Ph.D. dissertation, Faculty of Health, Engineering and Science, Victoria University, 2012. 